\title{
The Benefits of Plasma Cleaning for TKD/EBSD Analysis
}

Barbara Armbruster ${ }^{1}$, Michael Cable ${ }^{1}$, Ewa Kosmowska ${ }^{1}$, Kim Larsen ${ }^{2}$, Patrick Trimby ${ }^{2}$ and Ronald Vane $^{1}$

${ }^{1}$ XEI Scientific, Inc., Redwood City, California, United States, ${ }^{2}$ Oxford Instruments Nanoanalysis, High Wycombe, Bucks, England, United Kingdom

Plasma cleaners have been recognized as a necessary accessory for rapidly and effectively eliminating hydrocarbon contamination from vacuum chambers and samples [1]. Operating at turbopump pressures [2], the Evactron plasma radical source with a unique RF hollow cathode generates a low-temperature RF plasma to create oxygen radicals when air is the feed gas. The oxygen radicals combine with surface hydrocarbons to form gaseous phases which are removed by the pumping system. The benefits of plasma cleaning include faster pumpdown times [3], improved image quality of serial block-face SEM volumetric sets [4], prevention of hydrocarbon deposition during imaging [5] and no damage to EDS detectors or xray windows due to oxygen radical generation [6].

Electron backscatter diffraction (EBSD) and transmission Kikuchi diffraction (TKD) are scanning electron microscope techniques used to characterize the microstructures of crystalline materials on the micro- and nano-scale. Specimen preparation is critical to obtain good EBSD and TKD results since the diffraction patterns originate from very close to the sample surface: this necessitates a surface free from contamination and preparation-induced dislocations [7-9]. For the analysis of nanostructured materials, where the superior spatial resolution of TKD makes it the preferred technique, the small measurement step size and relatively weak diffracted signal make the accumulated electron dose (i.e. the electron dose per square micrometer) extremely high. This increases the likelihood of contamination and drift that, in turn, reduces the effectiveness of the technique.

To investigate the benefits of plasma cleaning for TKD experiments, a nanostructured Ni sample was analyzed by TKD, using a $4 \mathrm{~nm}$ step size and an analysis speed of approximately 66 patterns per second (using a CCD-based EBSD detector). In the first analysis, no plasma cleaning was carried out; in the second analysis, using exactly the same electron beam and EBSD detector settings, the sample was plasma cleaned within the microscope chamber for 5 minutes prior to the investigation. Figure 1a shows an Evactron E50, the most recent addition to the XEI Scientific product line, mounted on a SEM chamber port. For TKD experiments an electron-transparent sample is placed at a short working distance $(5-7 \mathrm{~mm})$ just above the top of the EBSD detector's phosphor screen (Figure 1b).

The results of the analysis without prior plasma cleaning are shown in Fig. 1c: after initially good indexing, contamination builds up and the pattern quality decreases rapidly, with a corresponding drop in indexing. In addition, noticeable sample drift occurs, causing an apparent stretching of the grain shapes. For the analysis following in-chamber plasma cleaning, as shown in Fig. 1d, there was no degradation of pattern quality and consistently good indexing throughout the analysis. Minor drift was observed, but an effective characterization of the microstructure was possible with minimal data processing. With the latest generation of higher sensitivity, fiber-optic coupled CMOS EBSD detector, plasma cleaning would also enable significantly higher speed and/or higher resolution analyses due to the relative improvement in the quality of the diffraction patterns and lack of surface degradation.

Plasma cleaning of the sample prior to TKD experiments revealed many advantages. The improvement in pattern quality and enhanced measurement efficiency is needed for extended experiments, which can include fast and multiple scans at the same sample position (e.g. for repeated analyses during in-situ 
heating or straining experiments). The advantage of plasma cleaning is recommended for all high resolution TKD experiments. Plasma cleaning the sample inside the microscope chamber is recommended since the chamber, detectors, and the holder are cleaned simultaneously.

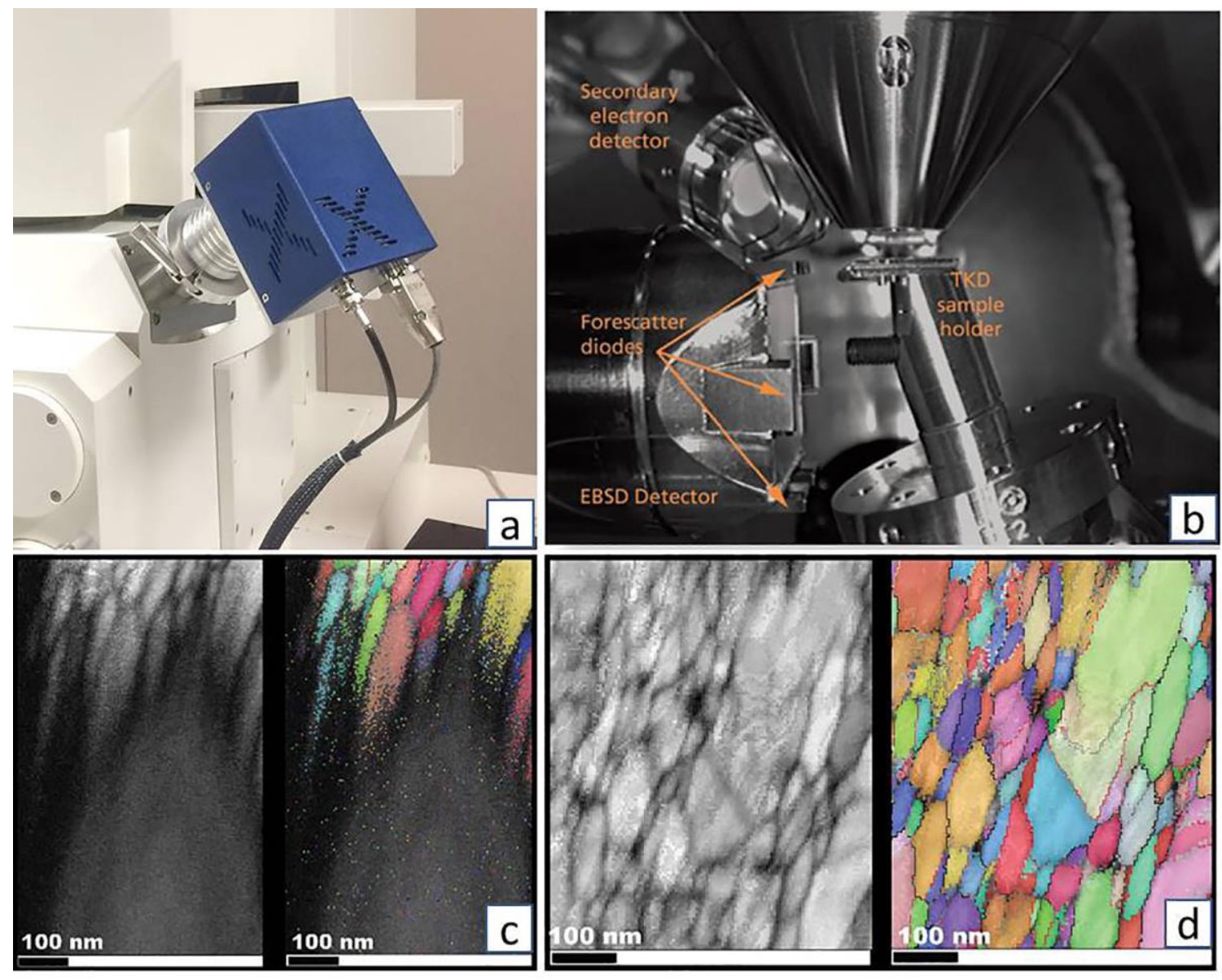

Figure 1. a) Evactron E50 on upper SEM chamber port, (b) sample geometry and detector positions for TKD experiments, (c) and (d) TKD pattern quality and inverse pole figure orientation maps of a nanostructured Ni Film: (c) without plasma cleaning; (d) after 5 minutes plasma cleaning. Note improved pattern quality, improved indexing and minimized drift in (d).

\section{References}

[1] R. Vane et al., Microsc. Microanal. 25 (S2) (2019), 550.

[2] R. Vane and M. Cable, Microsc. Microanal. 21 (S3) (2015), 161.

[3] E. Kosmowska et al., Microsc. Microanal. 23 (S1) (2017), 74.

[4] B. Armbruster et al., Microsc. Microanal. 23 (S1) (2017), 1266.

[5] B. Armbruster et al., Microsc. Microanal. 25 (S2) (2019), 540.

[6] E. Kosmowska et al., Microsc. Microanal. 25 (S2) (2019), 556.

[7] P. Trimby, Ultramicroscopy 120 (2012), 16.

[8] G. Sneddon et al., Mat. Sci. Eng. R. 110 (2016), 1.

[9] R. Borrajo-Pelaez \& P. Hedström, Crit. Rev. Solid State Mater. Sci, 43:6, (2018), 455. 